\title{
DNA methylation status of a distinctively different subset of genes is associated with each histologic Lauren classification subtype in early gastric carcinogenesis
}

\author{
YOSEP CHONG $^{1}$, KHALILULLAH MIA-JAN ${ }^{1}$, HOON RYU ${ }^{2}$, JAMSHID ABDUL-GHAFAR ${ }^{1}$, \\ JIJGEE MUNKHDELGER ${ }^{1}$, SAYAMAA LKHAGVADORJ ${ }^{1}$, SO YOUNG JUNG ${ }^{1}$, \\ MIRA LEE ${ }^{1}$, SUN-YOUNG JI ${ }^{1}$, EUNHEE $\mathrm{CHOI}^{3}$ and MEE-YON CHO ${ }^{1,4}$

\begin{abstract}
Departments of ${ }^{1}$ Pathology and ${ }^{2}$ Surgery, Yonsei University, Wonju College of Medicine; ${ }^{3}$ Division of Statistics, Institute of Life Style Medicine, Yonsei University, Wonju College of Medicine; ${ }^{4}$ Institute of Genomic Cohort, Yonsei University, Wonju College of Medicine, Wonju, Gangwon-do, Republic of Korea
\end{abstract}

Received December 31, 2013; Accepted February 26, 2014

DOI: $10.3892 /$ or.2014.3133

\begin{abstract}
DNA methylation change is known to play a crucial role in early gastric carcinogenesis. The present study aimed to identify and validate the correlation between differentially methylated regions (DMRs) and the subtypes of early gastric cancers (EGCs). Illumina Infinium methylation assay (IIMA; $450 \mathrm{~K}$ BeadChip kit) was performed on fresh tumor and non-tumor tissues of 12 EGCs to screen the methylation status of $450,000 \mathrm{CpG}$ sites. To evaluate the significance of DNA methylation in each histologic subtype, pyrosequencing assay (PA) was performed on 38 EGCs (18 intestinal-, 12 mixed- and 8 diffuse-type) using 12 genes selected from the screening. Between tumors of the intestinal-type $(n=6)$, and diffuse$(n=4)$ plus mixed-types $(n=2), 169$ regions showed significant differences (intensity $>3,000, \Delta \beta>0.2$ ) in IIMA. Hierarchical clustering using the 169 DMRs revealed distinct separation between the two groups. In PA using 12 selected genes from the IIMA results, the aberrant methylation statuses of DVL2 $(\mathrm{p}=0.0186)$ and $\operatorname{ETS} 1(\mathrm{p}=0.0222)$ were significantly related to diffuse- and mixed-types rather than the intestinal-type, while C19orf35 ( $p=0.019)$ and CNRIP1 $(p=0.0473)$ were related to the diffuse-type rather than intestinal-type, and GAL3ST2 $(p=0.0158)$ and ITGA3 $(p=0.0273)$ were related to the mixedtype rather than the other two types. The methylation of other genes, CLIP4, XKR6, CCDC57, MAML3 and SDC2, was related with age, tumor location, or Helicobacter infection rather than the histologic subtype. Aberrant DNA methylation of certain genes may be independently involved in each histo-
\end{abstract}

Correspondence to: Professor Mee-Yon Cho, Department of Pathology, Yonsei University, Wonju College of Medicine, No. 162 Ilsan-dong, Wonju, Gangwon-do, Republic of Korea E-mail: meeyon@yonsei.ac.kr

Key words: stomach neoplasm, DNA methylation, neoplasms by histologic type, genetic markers, stomach logic subtype of EGC. Furthermore, mixed-type EGCs may be a distinctive histologic subtype based on the different subset of DMRs compared to those of other subtypes.

\section{Introduction}

Despite its decreasing trend, gastric cancer (GC) still remains one of the most troublesome malignant diseases worldwide. It is the fourth most common cancer and the second leading cause of cancer-related death worldwide (1-3). In Asian countries such as Japan and Korea, it has the highest incidence among all malignancies although its overall survival rate has improved during the last few decades due to extensive screening programs $(3,4)$. Due to the poor prognosis and limited treatment options of advanced $\mathrm{GC}$, it remains a challenging task to determine effective molecular-pathological markers for early diagnosis with appropriate histological classification (5).

Lauren classification, which subgroups GCs mainly as two types, intestinal and diffuse, was first introduced in 1965 and has been generally accepted as a distinct and simple histological classification of GCs by most pathologists (6). Since the two major types show distinctively different phenotypic, epidemiologic and biologic characteristics, it is believed that different genetic alterations may be implicated in each histologic type of the classification (7). However, a considerable number of GCs accounting for $10-15 \%$ of cases, share the histologic features of these two types and are designated as mixed-type $(8,9)$. Although this type of tumor shows poorer prognosis than the common intestinal-type GCs, it is difficult to define and to diagnose when these tumors are encountered in a small biopsy. Furthermore, it is even more difficult to place this type of tumor into a specific category according to the WHO Classification of Gastric Tumours (2000) that was mainly founded on the Japanese Classification of Gastric Carcinoma (1981) and Lauren classification (9). For this reason, a new WHO classification has recently proposed (2010) this type of tumor as a mixed adenocarcinoma, while intestinaltype and diffuse-type are defined as tubular adenocarcinoma and poorly cohesive carcinoma, respectively (10). 


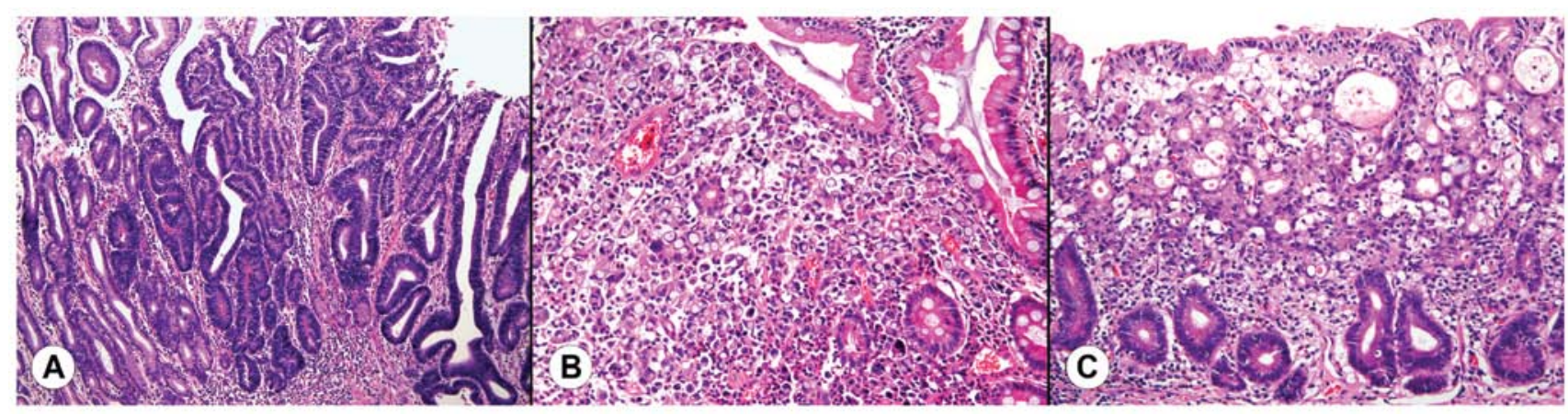

Figure 1. Histologic classification of early gastric carcinomas by Lauren classification: (A) intestinal-type, (B) diffuse-type and (C) mixed-type.

Considering that the tumor cells in diffuse-type cancers are loosely attached to each other from the early stage while intestinal-type cancers are derived from intestinal metaplasia and subsequent dysplasia, different key molecular changes may occur during the early steps of carcinogenesis in each type. For this reason, aberrant DNA methylation is being highlighted as the main change differentiating these subtypes of GCs from the very first stage $(1,11,12)$. Accumulating evidence from over 550 studies including the authors' previous study has demonstrated that aberrant epigenetic changes of more than 100 genes play a crucial role in the early steps of gastric carcinogenesis $(2,13)$. However, the results of these studies were based only on a small portion of GCs and at times were conflicting in regards to a certain candidate gene. That may be because most studies reported to date have focused on the presence of epigenetic alterations and their prognostic value as a screening tool without accurate consideration of their possible linkage with histologic or phenotypic features, and the stage of carcinogenesis that is mainly involved (2). Furthermore, large scale screenings of epigenetic changes in GCs have been rarely performed. Since various additional molecular changes as well as epigenetic alterations are involved throughout each stage of carcinogenesis, a careful research design is essential (11).

The present study aimed to identify key differential epigenetic changes that differentiate the histologic subtypes of GCs in the early steps of carcinogenesis. We performed a large scale screening of up to $485,000 \mathrm{CpG}$ sites with Illumina Infinium methylation assay (IIMA) and selected the most promising sites out of all the differentially methylated regions (DMRs) between the groups using one of the most reliable database, KEGG pathway database (14). We subsequently verified the results of the screening by pyrosequencing assay (PA).

\section{Materials and methods}

Patient samples. The present study was approved by the Institutional Review Board of Yonsei University, Wonju College of Medicine (GR-11-009). Written informed consent to participate in the present study was properly obtained for each subject. Surgically resected early gastric cancers (EGCs) from patients treated at the Wonju Severance Christian Hospital from 2011 to 2012 were prepared using the following process. First, fresh tissue from the tumors and adjacent corresponding non-tumor mucosa $(\mathrm{CNM})(0.5 \times 0.5 \mathrm{~cm}$-sized $)$ were collected from each case for the methylation study. Following routine formalin-fixed paraffin-embedded preparations and hematoxylin and eosin staining, full pathological evaluation with confirmatory histological classification according to Lauren were carried out for each case by three pathologists (Y. Chong, K. Mia-Jan and M.Y. Cho).

Diffuse-type GCs were defined when the tumor exclusively consisted of non-cohesive tumor cells diffusely infiltrating the stroma and exhibiting relatively deep infiltration with little or no gland formation (Fig. 1A). Intestinal-type GCs were defined when most part of the tumor consisted of recognizable glands similar to colonic or gastric mucosa (Fig. 1B). Poorly differentiated tumors that were difficult to categorize as either diffuse-type or mixed-type GCs were carefully excluded. Mixed-type GCs were defined when the tumor exhibited features of both typical intestinal-type and diffuse-type at the same time. In such cases, superficial parts of the tumor showed typical gland-like features as observed in intestinaltype GCs while the neck portion of the tumor consisted of irregular glands with non-cohesive singly scattered tumor cells with diffuse infiltration to the deeper part (Fig. 1C). Due to the histological similarity that mixed-type and diffusetype GCs share and the general proportion of each subtype among overall GCs, intestinal-type GCs were designated as group A, while mixed and diffuse-type $\mathrm{GC}$ were designated as group B. Presence of Helicobacter pylori (H. pylori) infection and intestinal metaplasia in adjacent CNM were also evaluated. Next, 38 cases of EGC and CNM including the previous 12 cases were also enrolled for validation of DMRs using PA.

DNA extraction and bisulfite conversion. DNAs for the following studies were extracted from each sample using DNeasy Blood and Tissue kit ${ }^{\circledR}$ (cat. no. 69506; Qiagen, Valencia, CA, USA), according to the manufacturer's instructions. The procedure included a proteinase $\mathrm{K}$ digestion at $55^{\circ} \mathrm{C}$ overnight. DNAs were quantified by NanoDrop ${ }^{\circledR} \mathrm{UV}-\mathrm{V}$ is spectrophotometer (NanoDrop Technologies, Wilmington, DE, USA) and diluted to $\sim 50 \mathrm{ng} / \mathrm{l}$ for IIMA. Eight hundred nanograms to $1 \mu \mathrm{g}$ of genomic DNAs were treated with sodium bisulfite using the EZ-96 DNA methylation kit ${ }^{\circledR}$ (cat. no. D5004; Zymo Research, Orange, CA, USA) according to the manufacturer's procedure.

DNA methylation profiling by the Infinium Human Methylation-450K BeadChip kit ${ }^{\circledR}$. The methylation assay was performed on $4 \mu \mathrm{l}$ bisulfite-converted genomic DNA (50 $\mathrm{ng} / \mu \mathrm{l}$ ) 


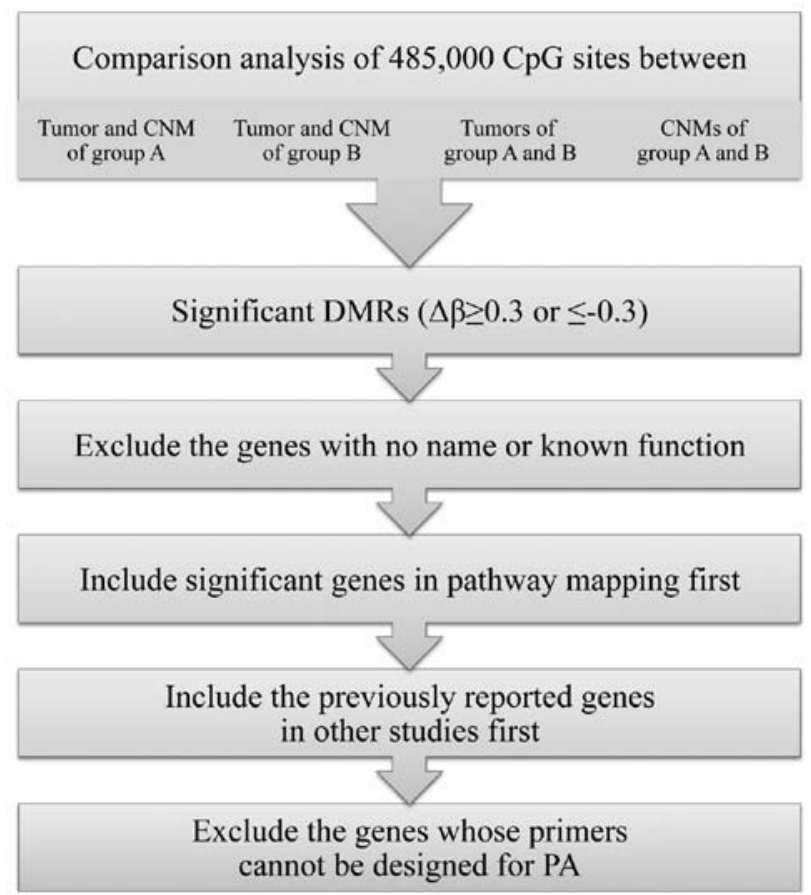

Figure 2. Determination of the eligible genes for pyrosequencing assay.

with Illumina HiScanSQ scanner (Illumina Inc., San Diego, CA, USA) according to the Infinium methylation assay protocol. Data quality was checked with the GenomeStudio ${ }^{\mathrm{TM}}$ for Methylation Module software (2010.3) and all samples passed this quality control. Uncorrected $\beta$-values were extracted with the same software. Raw data were submitted to the Gene Expression Omnibus (GPL8490) dataset.

To determine DMRs, the average $\beta$-values were computed with $\beta$-values when they had a signal intensity of $>3,000$. $\beta$-value is defined as the intensity of the methylated region over the sum of the intensities of the methylated and unmethylated regions plus 100, and a value of 0 designates a totally unmethylated status while the value 1 designates full methylation. $\Delta \beta$-value was calculated between two comparison groups by subtracting one $\beta$-value from the other (tumor vs. CNM in group A and B; CNM of group A vs. B and tumor of group A vs. B). When this value was $>0.2$ or $<-0.2$, it was considered to be meaningful. The results were considered to be significant when the p-value was $<0.05$. A differential score was used for evaluating the significance of the DMRs and when it was $>13$ or $<-13$, it was considered to have the same significance as a p-value of 0.05 , and when it was $>22$ or $<-22$, a p-value of 0.01 was indicated.

Validation by PA and determination of the eligible candidate genes. The steps that were used to select the most significant candidate genes eligible for PA are summarized in Fig. 2. Among the $\mathrm{CpG}$ sites that showed significant differential methylation between the compared groups in the hierarchical clustering of IIMA, the sites with high $\Delta \beta$-values $(>0.3$ or $<-0.3)$ were only selected. Next, the DMRs with no designation or known function were excluded. We then evaluated the potential functional role of selected DMRs by pathway mapping with DAVID Bioinformatics Resources 6.7 (http:// david.abcc.ncifcrf.gov/) using Kyoto Encyclopedia of Genes and Genomes (KEGG) pathway database (http://www.kegg. $\mathrm{jp} / \mathrm{kegg} /$ pathway.html). The pathways with most DMRs were considered to be significantly implicated with the DMRs discovered by IIMA, and the DMRs in those pathways were enrolled to the list of candidate genes first. Next, we reviewed the literature and included the genes that were previously reported in other studies. Subsequently, we excluded the genes from the list whose primers cannot be designed for PA due to the inappropriate $\mathrm{CG}$ content in the targeted sequence.

Eligibility for designing appropriate primers for PA was checked by PyroMark ${ }^{\circledR}$ Assay Design 2.0 software (Qiagen, Hilden, Germany). The designed primers were carefully validated for proper amplification after preliminary polymerase chain reaction (PCR) tests. One microliter of the converted DNA was used as a template in each subsequent PCR. PCRs were performed with HotStarTaq ${ }^{\circledR}$ Plus Master Mix kit (Qiagen, Hilden, Germany) under the following conditions: $95^{\circ} \mathrm{C}$ for $15 \mathrm{~min} ; 40$ cycles of $95^{\circ} \mathrm{C}$ for $1 \mathrm{~min} ; 50^{\circ} \mathrm{C}$ for $1 \mathrm{~min} ; 72^{\circ} \mathrm{C}$ for $1 \mathrm{~min}$ and $72^{\circ} \mathrm{C}$ for $10 \mathrm{~min}$. The success of the amplification was assessed with $2 \%$ agarose gel electrophoresis. Pyrosequencing of the PCR products was performed with the PyroMark ${ }^{\mathrm{TM}}$ Q24 system (Qiagen, Uppsala, Sweden) according to the manufacturer's instructions. Blue values (perfect calls) and yellow values (needed to be checked manually) were considered for subsequent analyses.

Statistical analyses. After initial processing of the raw data of IIMA, general statistical computing was carried out by $\mathrm{R}$ project. For hierarchical clustering analysis, correlation, Euclidean and Manhattan methods were applied. To search any possible correlations within the data, Pearson's correlation method was utilized.

For the PA, univariate analysis using paired t-test, correlation test, two-sample t-test, one-way ANOVA on the differences of methylation status were used for comparing the results according to Lauren classification (intestinal, diffuse and mixed, and group A and B), location (proximal and distal), age and presence of $\mathrm{H}$. pylori. For hierarchical clustering analysis, correlation, Euclidean or Manhattan methods were applied as in the IIMA. To search for any possible correlations within the data, Pearson's correlation method was utilized. Scheffe's method for multiple comparisons was used to validate the genes that were found to have significant differences between any of the two types of the Lauren classification.

\section{Results}

Samples used for analysis. The 12 cases of EGC analyzed using IIMA consisted of 6 cases of intestinal-type, 4 cases of diffuse-type and 2 cases of mixed-type (M:F, 3:1; age range 44-77 years; median age, 60).

Thirty-eight cases of EGC used for the PA consisted of 19 cases of intestinal-type, 8 cases of diffuse-type and 11 cases of mixed-type (M:F, 3.75:1; age range 31-82 years; median age, 67).

\section{DNA methylation profiling by IIMA}

Hierarchical clustering analysis. Upon hierarchical clustering analysis of 6 cases of group A tumors and matched CNM, no 
A

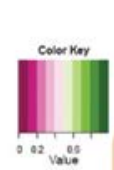
$\begin{array}{cc}\text { Normal of } & \text { Normal of } \\ \text { Intestinal-type } & \text { Diffuse \& Mixed-type }\end{array}$ (Group A) (Group B)

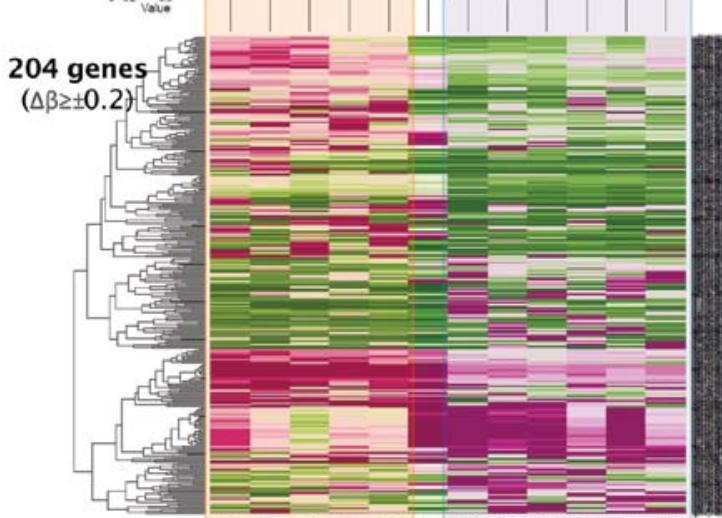

N2 N4 N3 N5 N1 N6 N12 N9 N8 N10N11 N7
B

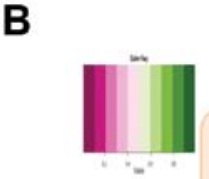
Tumors of
Intestinal-typ $\begin{array}{cc}\text { Intestinal-type } & \text { Diffuse \& Mixed- } \\ \text { (Group A) } & \text { (Group B) }\end{array}$ Tumors of

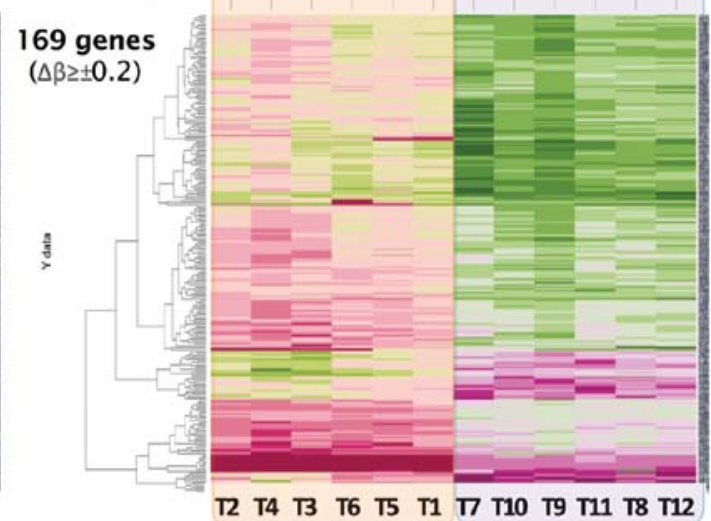

Figure 3. Hierarchical clustering analysis of IIMA. (A) In the normal tissues, a distinct grouping between group A (intestinal-type) and B (diffuse-type and mixed-type) was found with the DNA methylation patterns of 208 genes except N6 (statistically not significant). (B) In the tumors, a statistically significant distinct grouping between group A and B was found in the DNA methylation patterns of 169 genes. IIMA, Illumina Infinium methylation assay.

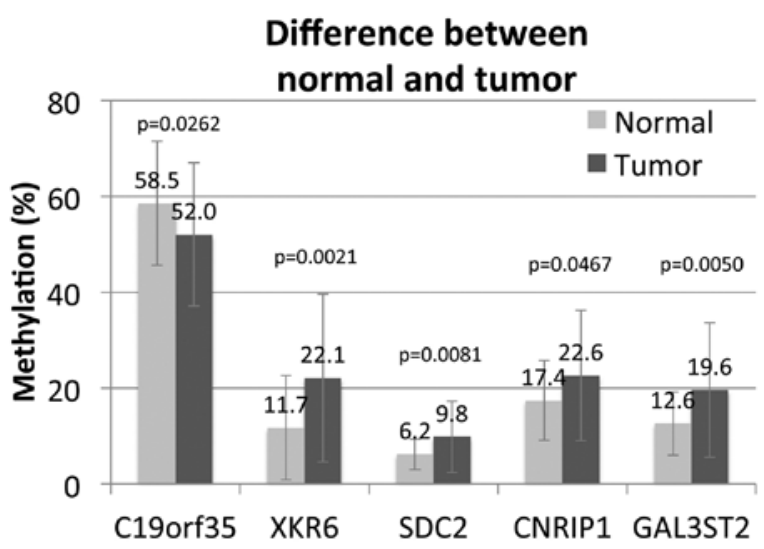

Figure 4. Methylation differences in 5 significant genes between overall normal and tumor tissues.

distinct grouping was found with DMRs by either correlation, Euclidean or Manhattan methods. Between the tumors and CNM of group B, on the other hand, a clear grouping was made by 208 genes in 3 cases, 2 cases being mixed-type, by both correlation and Euclidean methods (T7, T11 and T12 vs. N7, $\mathrm{N} 11$ and N12). However, it seemed inappropriate to evaluate the significance of this grouping since half of the cases were not concordantly grouped with the other 3 cases.

Hierarchical clustering analysis of CNM showed a distinct separation between group A and B (except N6) (Fig. 3A). Although it was not statistically significant, 204 genes showed a differential methylation status between the two groups with $\Delta \beta$-value of $>0.2$. The hierarchical clustering analysis of the tumors of group A and B showed a distinct grouping with 169 statistically significant DMRs ( $\Delta \beta$-value $\geq \pm 0.2$, differential score $\geq 13, p<0.05$ ) (Fig. 3B). In total, $>800$ DMRs were found to be significantly different with a $\Delta \beta$-value of $>0.2$ or $<-0.2$ between the subgroups. After removal of the DMRs without a specific gene name or with an unspecified function,
581 genes were pooled as candidates (208 genes between tumors and CNM in group B, 204 genes found between CNM of group A and B, and 169 genes between tumors of group A and B). Among these, 197 genes were included in the list of the candidate genes with $\Delta \beta$-value $>0.3$ or $<-0.3$.

\section{Validation of ascertained CpGs by $P A$}

Determination of the genes eligible for PA. As a result of pathway mapping by KEGG pathway database, 18 genes out of 197 genes were found to be significantly related to the archived pathways (pathways in cancers, Notch-signaling pathway, dorso-ventral axis formation, $\mathrm{p}<0.05)$. Five genes, $A P C$, $D V L 2, E T S 1, I T G A 3$ and MAML3, among them were eligible for designing primers for PA and showed a robust amplification in a pilot study. An additional 7 genes from the genes with top $\triangle \beta$-values, C19orf35, XKR6, CLIP4, SDC2, CNRIP1, GAL3ST2 and CCDC57, were selected after checking for primer eligibility and showed a robust amplification in the pilot study. As a result, finally 12 genes from the screening result were determined as eligible for producing tailored primers for the following PA. Basic information of these 12 genes is listed in Table I. Each gene includes one or more (up to 8) CpG sites.

Genes with significantly differential methylation statuses between tumors and CNMs. Univariate analysis showed significant differences in the methylation status between all tumors and their CNM regardless of type for 5 genes: C19orf35, XKR6, SDC2, CNRIP1 and GAL3ST2 ( $\mathrm{p}=0.0262$, $0.0021,0.0081,0.0467$ and 0.0050, respectively) (Fig. 4). Four of the genes showed hypermethylation in the tumor tissue when compared to the CNM (XKR6, SDC2, CNRIP1 and GAL3ST2) with an average of 10.4, 3.6, 5.2 and 7.0, respectively, while C19orf35 was significantly hypomethylated in the tumor tissue when compared to the CNM (average -6.5).

Significantly differential methylation statuses according to Lauren classification. ANOVA revealed a statistical 
Table I. Primer sequences used in the pyrosequencing assay.

\begin{tabular}{|c|c|c|c|c|c|}
\hline Gene & Ch. & & Sequences & $\begin{array}{l}\text { Amplicon } \\
\text { size (bp) }\end{array}$ & CpGs \\
\hline C19orf35 & 19 & $\begin{array}{l}\text { F- } \\
\text { R- }\end{array}$ & $\begin{array}{l}\text { AGATGGGGTATTGGGGATATTTTA } \\
\text { CCTCCTAAACAACTCACCC }\end{array}$ & 146 & 8 \\
\hline XKR6 & 8 & $\begin{array}{l}\text { F- } \\
\text { R- }\end{array}$ & $\begin{array}{l}\text { GGGGTTTTTAGTTTAGTTGG } \\
\text { TCCСТACССССТTCCACTA }\end{array}$ & 158 & 8 \\
\hline$C L I P 4^{\mathrm{a}}$ & 2 & $\begin{array}{l}\text { F- } \\
\text { R- }\end{array}$ & $\begin{array}{l}\text { GGGGTGTYGTAGTGTTATTTT } \\
\text { CTAAATCCCCYACCCCAACAACT }\end{array}$ & 146 & 7 \\
\hline$S D C 2^{\mathrm{a}}$ & 8 & $\begin{array}{l}\text { F- } \\
\text { R- }\end{array}$ & $\begin{array}{l}\text { GGAGGYGGTAGTGTGATTTTTAGAT } \\
\text { AACYCAAAAACCCTAACTTACC }\end{array}$ & 148 & 6 \\
\hline$C N R I P 1^{\mathrm{a}}$ & 2 & $\begin{array}{l}\text { F- } \\
\text { R- }\end{array}$ & $\begin{array}{l}\text { GGAGGAGTYGGTGAGTAGTT } \\
\text { CCAAACCCTCYCCCAAACATA }\end{array}$ & 153 & 6 \\
\hline GAL3ST2 & 2 & $\begin{array}{l}\text { F- } \\
\text { R- }\end{array}$ & $\begin{array}{l}\text { GTGTGTTAGAGGTTTGTGATG } \\
\text { CCAAAAACCCTACAAAAATCCCC }\end{array}$ & 196 & 6 \\
\hline$C C D C 57$ & 17 & $\begin{array}{l}\text { F- } \\
\text { R- }\end{array}$ & $\begin{array}{l}\text { TAGATATTGTTGTTGGGAGTAGTT } \\
\text { TCATTTAATCATTCTCTCCTTCAT }\end{array}$ & 227 & 3 \\
\hline$A P C$ & 5 & $\begin{array}{l}\text { F- } \\
\text { R- }\end{array}$ & $\begin{array}{l}\text { ATATGTGGTTGTATTGGTG } \\
\text { ACATACTACAATAAACCCTATACCA }\end{array}$ & 194 & 3 \\
\hline$D V L 2$ & & $\begin{array}{l}\text { F- } \\
\text { R- }\end{array}$ & $\begin{array}{l}\text { GTTTAGGGTAGAGATAGTTTGATTG } \\
\text { ACTATACACTAAAACTCCCCTATC }\end{array}$ & 180 & 2 \\
\hline ETS1 & 11 & $\begin{array}{l}\text { F- } \\
\text { R- }\end{array}$ & $\begin{array}{l}\text { ATTTTTTAATGTTAAGGGGAGATGG } \\
\text { ATCTCСТTACCСTCAACC }\end{array}$ & 205 & 4 \\
\hline ITGA3 & 17 & $\begin{array}{l}\text { F- } \\
\text { R- }\end{array}$ & $\begin{array}{l}\text { GATTTTGTGGGTTTATGGGGATAT } \\
\text { CTTAAAAACTACCAAACACCTACTT }\end{array}$ & 201 & 1 \\
\hline$M A M L 3$ & 4 & $\begin{array}{l}\text { F- } \\
\text { R- }\end{array}$ & $\begin{array}{l}\text { GGAGTTTTTTGAGGATTTGTAAGTA } \\
\text { AATTCCAAAACCCCAACTACA }\end{array}$ & 219 & 7 \\
\hline
\end{tabular}

Ch., chromosome number. ${ }^{2} Y$ designates $\mathrm{CpG}$ sites included in the primer sequences.

difference in methylation status according to the Lauren classification in tumor tissues (C19orf35, CNRIP1 and ITGA3; $\mathrm{p}=0.0192,0.0373$ and 0.0146 , respectively) and matched CNM (GAL3ST2, DVL2, ETS1 and ITGA3; p=0.0157, 0.0182, 0.0099 and 0.0259, respectively) (Fig. 5). In Scheffe's method of multiple comparisons, C19orf35, CNRIP1 and DVL2 were significantly different between the diffuse-type and intestinal-type ( $\mathrm{p}=0.0195,0.0473$ and 0.0186 , respectively) while GAL3ST2, ETS1 and ITGA3 were different between the mixed-type and intestinal-type GCs $(\mathrm{p}=0.0158,0.0222$ and 0.0273 , respectively). Integrated interpretation of the methylation pattern of these 6 genes according to histologic subtype allowed us insight that three groups, DVL2 and ETS1, C19orf35 and CNRIP, GAL3ST2 and ITGA3, present different transition between tumors and matched CNM. DVL2 and ETS1 were hypomethylated both in the tumor tissue and the CNM of intestinal-type EGCs while they were hypermethylated in the mixed-type and diffuse-type EGCs (Fig. 5A and B). On the other hand, the methylation statuses of C19orf35 and CNRIP in the three histologic subtypes exhibited a similar level in the CNM while those varied in the tumors according to histologic subtype (Fig. 5C and D). Notably, GAL3ST2 and
ITGA3 were significantly hypermethylated in the tumors and the CNM of the mixed-type rather than in the intestinal-type and diffuse-type subgroups (Fig. 5E and F).

The hierarchical clustering analysis using the methylation status of these 6 genes showed a relatively distinct grouping between each type as the results of the hierarchical clustering analysis using IIMA (Fig. 6).

Relationship between genes with significantly differential methylation status and patient age, tissue location and $H$. pylori infection. To determine whether changes in methylation status are related with non-tumor factors, we statistically analyzed the correlation between methylation status in the non-neoplastic mucosa with patient age, location and $H$. pylori infection (15). There was a significant correlation between patient age and the methylation status of CLIP4 (Fig. 7A). Paired t-test revealed a significant difference in the methylation in C19orf35, XKR6, CLIP4, CNRIP1, CCDC57 and MAML3 between proximal and distal location $(\mathrm{p}=0.0002,0.0088$, $0.0038,0.0003,0.0317$ and 0.0203 , respectively; average difference, -14.6, 8.0, 10.5, 8.6 and 8.3, respectively) (Fig. 7B). The methylation level of all genes except that of C19orf35 
A

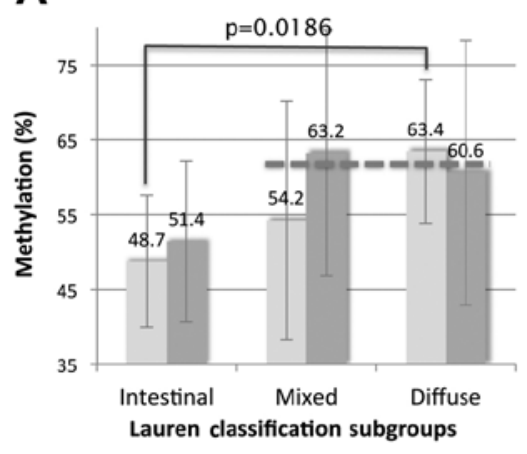

B

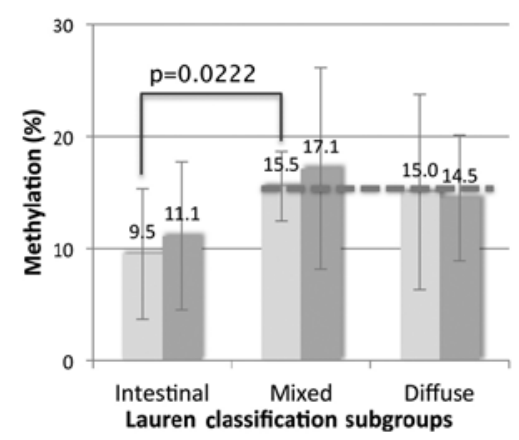

C

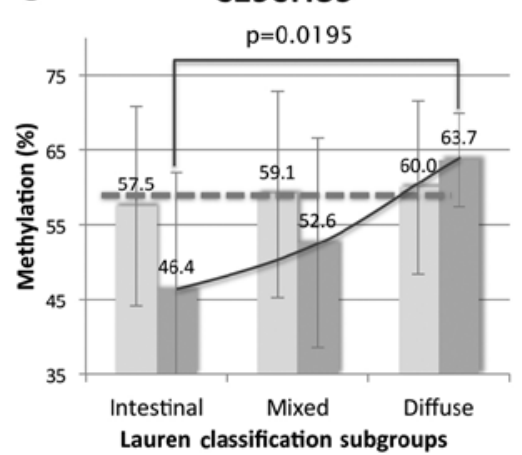

D

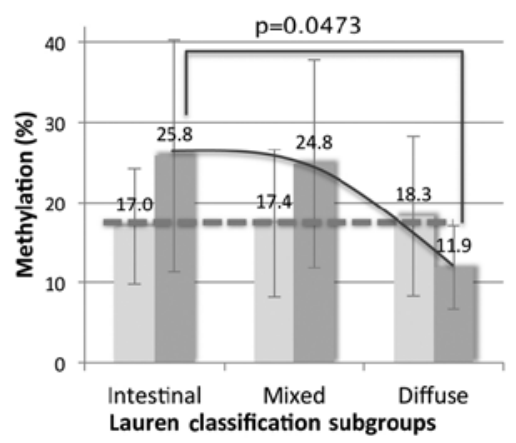

E

GAL3ST2

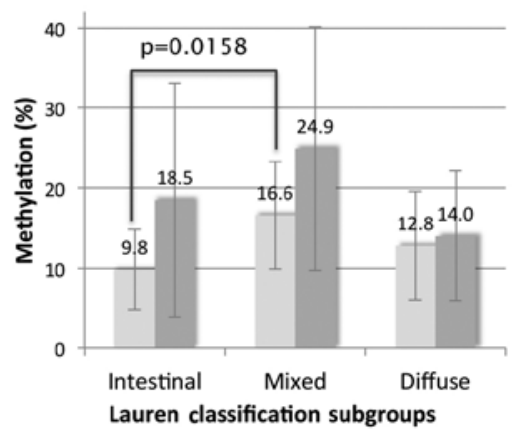

$\mathbf{F}$

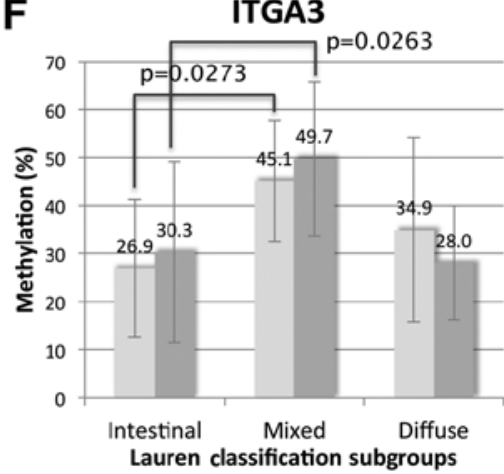

Figure 5. Differences in methylation among the three Lauren classification subtypes. Light gray bar, normal; dark gray bar, tumor tissue.

was significantly higher in the distal compared to proximal stomach. However, no significant difference was found in the tumor tissues according to their location.

In the one-way ANOVA, SDC2 was significantly hypermethylated in CNM with $H$. pylori infection $(\mathrm{p}=0.0056$, average difference, 4.4) (Fig. 7C). Aberrant methylation of $S D C 2$ was found to correlate with diffuse-type and mixedtype GCs (Fig. 7D and E).

\section{Discussion}

Based on methylation analysis using IIMA validated by PA, we indirectly verified that certain methylation changes are closely associated with the carcinogenesis of GC. Moreover, different subsets of aberrantly methylated genes are involved in the carcinogenesis of a specific histologic subtype of GC, logically suggesting that mixed-type GC may be a distinct subtype. Hierarchical clustering analysis of IIMA was broadly concordant with the results of PA. Tumors of group A and B in IIMA showed a sharply demarcated distinction in the methylation pattern for 169 genes (Fig. 3B). A rather clear grouping of 208 genes in 3 cases of group B indirectly denoted the molecular uniqueness of mixed-type GCs, although the data should be interpreted with extreme caution due to the limited number of cases and incomplete grouping.

Based on an integrated interpretation of the statistical analysis of the results of PA, we classified the genes with their methylation patterns according to the histologic subtypes into three groups: i) DVL2 and ETS1, ii) C19orf35 and CNRIP1, and iii) GAL3ST2 and ITGA3 (Fig. 5).
DVL2 and ETS1 were specific to diffuse-type and mixedtype compared to the intestinal-type subgroup. Both genes showed a similar methylation pattern both in the CNM and in the tumors of the intestinal-type while they showed significantly higher methylation in the CNM as well as in the tumors of the mixed-type and diffuse-type rather than the intestinaltype (Fig. 5A and B). These results are consistent with previous findings that some aberrantly methylated genes in tumors of certain subtypes are also aberrantly methylated in CNM (16). This suggests that the aberrant methylation of these two genes may start from the CNM exclusively in mixed-type and diffusetype subgroups. Similarly to these results, these genes showed significant difference in methylation in the CNM between group A and B in the hierarchical clustering analysis of IIMA. Dishevelled, symbolized as DVL2, is a positive mediator of Wnt/ $\beta$-catenin signaling and its aberrant overexpression has been known to result in neoplastic transformation, yet the direct involvement in human cancer has not been reported to date (17). Since the function of Wnt signaling components in gastric carcinogenesis has been recently described, it seems advisable to evaluate the methylation change of this gene in relation to other components of Wnt signaling. Contrary to our screening results and the accumulated evidence on the aberrant methylation of $A P C$ in GC, we could not find any connection between $A P C$ and the parameters examined (16). The ETS1 proto-oncoprotein is a member of the Ets family of transcription factors and has been implicated in invasive behavior in relation to angiogenesis in many types of human cancer such as astrocytomas, breast cancer and some types of colon cancer (18). It is not expressed in normal gastric epithe- 


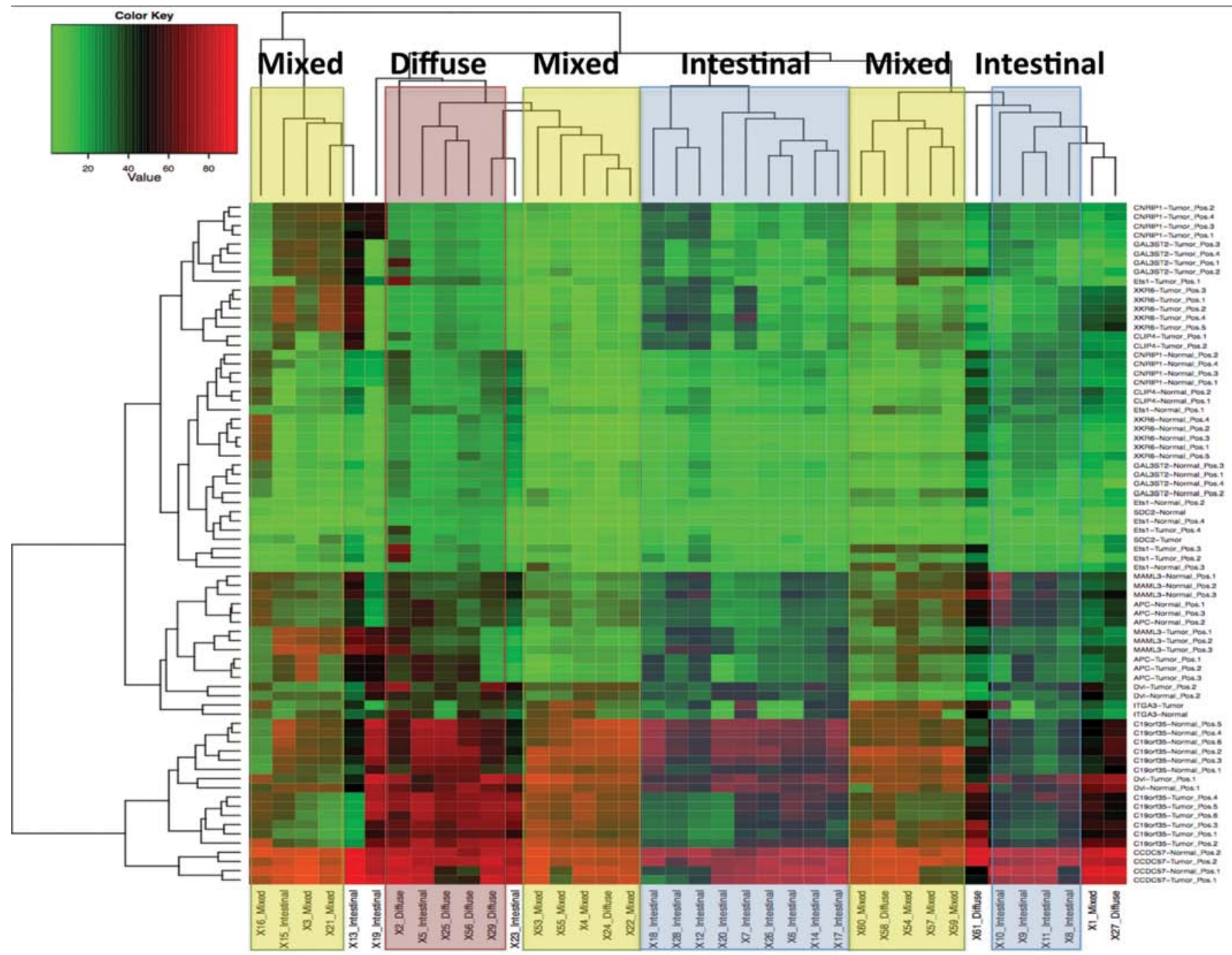

Figure 6. Hierarchical clustering analysis of the pyrosequencing assay.

lium and is overexpressed in $\mathrm{GC}$, resulting in its value as a marker of poor prognosis (18-20). To our knowledge, no information exists to date on the methylation change of this gene in GC. Further evaluation for comprehensive understanding is needed.

C19orf35 and CNRIP1 were specific to diffuse-type compared to the intestinal-type subgroups. Both revealed a similar level of methylation in CNM regardless of the histologic subtype of tumor while the methylation of the tumor tissue varied with the histologic type (Fig. 5C and D). C19orf35 was hypermethylated but $C N R I P 1$ was hypomethylated in diffusetype tumors compared to the CNM while it had an opposite trend in the tumors of the intestinal-type and mixed-type subgroups. Importantly, these findings suggest the possibility of utilizing these genes as specific markers for diffuse-type GCs. On the other hand, the function of C19orf35 is still unknown while frequent methylation of $C N R I P 1$ has recently been reported in colorectal cancers and adenoma $(21,22)$.

GAL3ST2 and ITGA3 were specific to mixed-type compared to intestinal-type and diffuse-type subgroups. They were specifically hypermethylated in CNM as well as in the tumor tissue of mixed-type GC (Fig. 5E and F). Thus, this analogously represents the molecular unique- ness of mixed-type GCs contrary to the idea that these are intermingled tumors of intestinal-type and diffuse-type GCs. Hierarchical clustering of IIMA also showed a distinctive methylation pattern of mixed-type compared to diffuse-type GCs, although the data were not perfect. Little is known about the functional role of GAL3ST2, a member of the galactose-3- $O$-sulfotransferase protein family, in human types of cancers. However, a recent series of evidence indicates that downregulation of GAL3ST2 expression is observed in colon cancers, and GAL3ST2 has also been implicated in the metastatic potential of breast and laryngeal cancers (23-25). Considering the mounting evidence on the central role of selectins such as galectin-3 in thyroid, pancreatic, colon and even GCs in relation to several relevant genes, the associative function of Gal:3- $O$-sulfotransferase in GCs should be emphasized and evaluated in more detail (26-29). For ITGA3, it has been established that $\alpha 3 \beta 1$ integrin is involved in the maintenance of basement membrane integrity through adhesion to laminin 5 and plays an essential role in the invasiveness and metastatic potential of tumor cells (30-32). However, there are contradictory reports regarding the significance of ITGA3 expression in various types of cancers (33-36). Considering the results demonstrated recently that GAL3ST3 is involved 
A Methylation of CLIP4 in normal tissue according to age

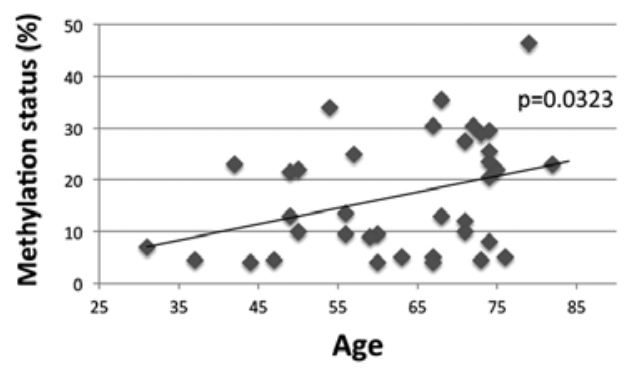

SDC2 in comparison of

C SDC2 according to $H$. pylori infection

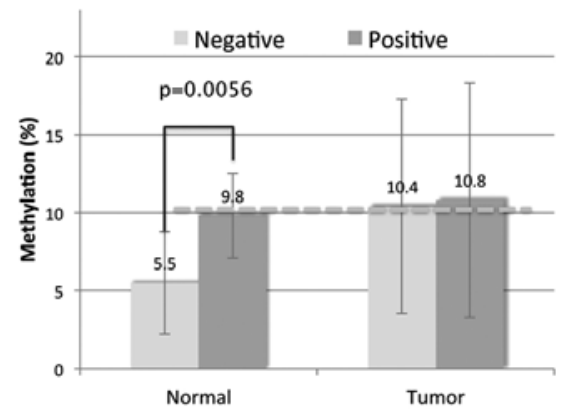

B

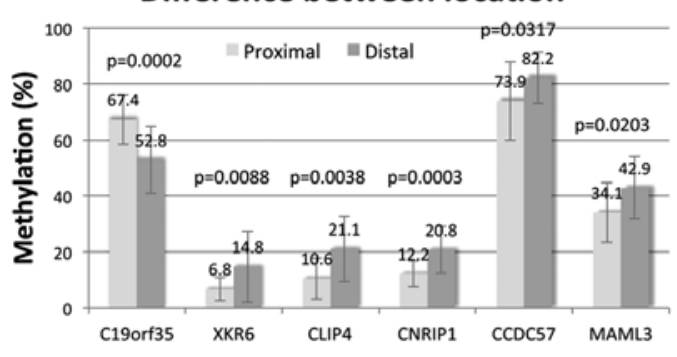

Figure 7. Methylation of various genes in relation to age, location and $H$. pylori infection in normal tissues. (A) The methylation of $C L I P 4$ in the normal tissues increased with the age of the GC patients in the correlation analysis. (B) The methylation of six genes, C19orf35, XKR6, CLIP4, CNRIP1, CCDC57 and $M A M L 3$, in the normal tissues significantly varied according to tumor location. (C) SDC2 exhibited significant hypermethylation in CNM with H. pylori infection. (D and E) Aberrant methylation of $S D C 2$ was found to correlate with diffuse-type and mixed-type GC.

in tumor metastasis by regulating the ability of adhesion to selectins and expression of integrins, more evidence is needed to clarify the relationship among these molecules (25). A recent study by Park et al strengthens the results of the present study by showing significantly higher frequencies of $\mathrm{CpG}$ island hypermethylation of 16 genes in mixed-type GCs when compared to diffuse-type and intestinal-type subgroups (37). They found no difference in the number of methylated genes between diffuse-type and intestinal-type-like components of mixed-type cancers, rejecting the hypothesis that mixed-type GCs may be the mixture of intestinal-type and diffuse-type GCs.

The heatmap made with the pyrosequencing data of the aforementioned 6 genes also demonstrated a rather clear distinction between each subtype (Fig. 6).

Regarding the results of $S D C 2$ methylation in relation to $H$. pylori infection, this hypothesis seems to be more credible since $S D C 2$ in $C N M$ with $H$. pylori infection revealed significant hypermethylation compared to CNM without H. pylori infection up to the level of tumor regardless of H. pylori infection (Fig. 7C). These results are in line with the results of previous studies that some genes such as $\mathrm{CDH}$, known for having diffuse-type specificity, are hypermethylated in cases with $H$. pylori infection $(12,16)$. These indirectly suggest the possible pathogenic relationship between the genes and $H$. pylori infection as well as between $H$. pylori infection and certain histologic types of GC. In our results, aberrant methylation of $S D C 2$ was found to correlate with diffuse-type and mixed-type GC (Fig. 7D and E). Recently, a series of genes have been implicated in gastric carcinogenesis in rela- tion to $H$. pylori infection (38-43). However, $S D C 2$ was not included in the list. $S D C 2$ is known as one of a four-member family of transmembrane heparin sulfate proteoglycans and plays important roles in many human types of cancers such as colorectal, hepatic and pancreatic cancers $(44,45)$. The function of $S D C 2$ in the gastric carcinogenesis of GC is still unclear. Interestingly, a recent study raised the possibility that upregulation of $S D C 2$ may play an important role in peritoneal metastasis of human GC cells (46). Taken together, these results emphasize the importance of molecular assessment of premalignant or even non-neoplastic mucosa of stomach in the presence of $H$. pylori infection.

Even in the non-tumor tissues, the methylation status of certain genes was affected by various factors such as age, location and the status of $H$. pylori infection similar to the results of previous studies (16). Similar to CLIP4 in the present study, other genes have also been documented to show a general progressive increase in methylation in normal gastric tissues as the age of the patient increases (16). Six genes showed significantly differential methylation by PA between the anatomic locations (Fig. 7B). Considering that the component of gastric mucosa varies with location, a differential methylation status of genes according to location in normal tissues may be justifiable. Similar results in previous studies have demonstrated differences in the methylation status of genes in non-neoplastic tissues of different organs or different sites in an organ (15).

XKR6 showed significant hypermethylation in the CNM of the distal stomach $(\mathrm{p}=0.0009)$ as well as in overall tumor tissues $(p=0.008)$. This suggests that certain methylation changes may be involved in making specific locations of 
the stomach susceptible for carcinogenesis. Recent studies have reported that the methylation statuses of certain genes such as $C D K N 2 A, C O X-2$ and $H M L H 1$ are significantly different according to tumor location $(38,39)$. Little is known concerning XKR6 in human disease except that it is a member of the Kell blood group complex subunit-related family and its SNP (rs6985109) may be a potential risk factor in systemic lupus erythematosus (47).

The evaluation of the methylation status of certain genes may be helpful to distinguish between the histologic subtype of EGC. The methylation statuses of DVL2, ETS1, C19orf35, CNRIP, GAL3ST2 and ITGA3 were significantly different according to the histologic subtype of the EGC. We found that the methylation patterns of GAL3ST2 and ITGA3 were specifically different in mixed-type compared to the intestinal-type or diffuse-type subgroups, suggesting that the mixed-type is a distinct histologic subtype. The methylation of various genes that are associated with non-tumor factors such as age, location and $H$. pylori infection should be considered.

\section{Acknowledgements}

This study was supported by a Korea Science and Engineering Foundation (KOSEF) grant funded by the Korean government (MEST) (grant code: 2012-8-5113).

\section{References}

1. Dicken BJ, Bigam DL, Cass C, Mackey JR, Joy AA and Hamilton SM: Gastric adenocarcinoma: review and considerations for future directions. Ann Surg 241: 27-39, 2005.

2. Sapari NS, Loh M, Vaithilingam A and Soong R: Clinical potential of DNA methylation in gastric cancer: a meta-analysis. PLoS One 7: e36275, 2012.

3. Shin A, Kim J and Park S: Gastric cancer epidemiology in Korea. J Gastric Cancer 11: 135-140, 2011.

4. Inoue $M$ and Tsugane S: Epidemiology of gastric cancer in Japan. Postgrad Med J 81: 419-424, 2005.

5. Yasui W, Oue N, Aung PP, Matsumura S, Shutoh M and Nakayama H: Molecular-pathological prognostic factors of gastric cancer: a review. Gastric Cancer 8: 86-94, 2005.

6. Lauren P: The two histological main types of gastric carcinoma: diffuse and so-called intestinal-type carcinoma. An attempt at a histo-clinical classification. Acta Pathol Microbiol Scand 64: 31-49, 1965.

7. Tan IB, Ivanova T, Lim KH, et al: Intrinsic subtypes of gastric cancer, based on gene expression pattern, predict survival and respond differently to chemotherapy. Gastroenterology 141 476-485, 2011

8. Zheng HC, Li XH, Hara T, et al: Mixed-type gastric carcinomas exhibit more aggressive features and indicate the histogenesis of carcinomas. Virchows Arch 452: 525-534, 2008.

9. Ackerman LV and Rosai J: Rosai and Ackerman's Surgical Pathology. 9th edition. Mosby, St. Louis, MO, 2004.

10. Bosman FT and World Health Organization International Agency for Research on Cancer: WHO Classification of Tumors of the Digestive System. International Agency for Research on Cancer. 4th edition. IARC Press, Lyon, 2010.

11. Oue N, Mitani Y, Motoshita J, et al: Accumulation of DNA methylation is associated with tumor stage in gastric cancer. Cancer 106: 1250-1259, 2006.

12. Yamamoto E, Suzuki H, Takamaru H, Yamamoto H, Toyota $M$ and Shinomura Y: Role of DNA methylation in the development of diffuse-type gastric cancer. Digestion 83: 241-249, 2011.

13. Choi J, Cho MY, Jung SY, Jan KM and Kim HS: CpG island methylation according to the histologic patterns of early gastric adenocarcinoma. Korean J Pathol 45: 469-476, 2011.

14. Dedeurwaerder S, Defrance M, Calonne E, Denis H, Sotiriou C and Fuks F: Evaluation of the Infinium Methylation $450 \mathrm{~K}$ technology. Epigenomics 3: 771-784, 2011.
15. Hong SJ, Kang MI, Oh JH, et al: DNA methylation and expression patterns of key tissue-specific genes in adult stem cells and stomach tissues. J Korean Med Sci 24: 918-929, 2009.

16. Zhao $\mathrm{C}$ and $\mathrm{Bu} \mathrm{X}$ : Promoter methylation of tumor-related genes in gastric carcinogenesis. Histol Histopathol 27: 1271-1282, 2012.

17. Polakis P: Wnt signaling and cancer. Genes Dev 14: 1837-1851, 2000.

18. Dittmer J: The biology of the Ets1 proto-oncogene. Mol Cancer 2: 29, 2003.

19. Tsutsumi S, Kuwano H, Nagashima N, Shimura $T$, Mochiki E and Asao T: Ets-1 expression in gastric cancer. Hepatogastroenterology 52: 654-656, 2005.

20. Yu Y, Zhang YC, Zhang WZ, et al: Ets1 as a marker of malignant potential in gastric carcinoma. World J Gastroenterol 9: 2154-2159, 2003.

21. Lind GE, Danielsen SA, Ahlquist T, et al: Identification of an epigenetic biomarker panel with high sensitivity and specificity for colorectal cancer and adenomas. Mol Cancer 10: 85, 2011.

22. Oster B, Thorsen K, Lamy P, et al: Identification and validation of highly frequent $\mathrm{CpG}$ island hypermethylation in colorectal adenomas and carcinomas. Int J Cancer 129: 2855-2866, 2011.

23. Chandrasekaran EV, Lakhaman SS, Chawda R, Piskorz CF, Neelamegham S and Matta KL: Identification of physiologically relevant substrates for cloned Gal: 3-O-sulfotransferases (Gal3STs): distinct high affinity of Gal3ST-2 and LS180 sulfotransferase for the globo $\mathrm{H}$ backbone, Gal3ST-3 for $\mathrm{N}$-glycan multiterminal Gal $\beta 1$, 4 GlcNAc $\beta$ units and 6-sulfoGal $\beta 1$, $4 \mathrm{GlcNAc} \beta$, and Gal3ST-4 for the mucin core-2 trisaccharide. J Biol Chem 279: 10032-10041, 2004.

24. Seko A, Nagata K, Yonezawa S and Yamashita K: Downregulation of Gal 3-O-sulfotransferase-2 (Gal3ST-2) expression in human colonic non-mucinous adenocarcinoma. Jpn J Cancer Res 93: 507-515, 2002.

25. Shi BZ, Hu P, Geng F, He PJ and Wu XZ: Gal3ST-2 involved in tumor metastasis process by regulation of adhesion ability to selectins and expression of integrins. Biochem Biophys Res Commun 332: 934-940, 2005.

26. Cheong TC, Shin JY and Chun KH: Silencing of galectin-3 changes the gene expression and augments the sensitivity of gastric cancer cells to chemotherapeutic agents. Cancer Sci 101: 94-102, 2010.

27. Kim SJ, Choi IJ, Cheong TC, et al: Galectin-3 increases gastric cancer cell motility by up-regulating fascin-1 expression. Gastroenterology 138: 1035-1045, 2010.

28. Kim SJ, Shin JY, Lee KD, et al: Galectin-3 facilitates cell motility in gastric cancer by up-regulating protease-activated receptor-1 (PAR-1) and matrix metalloproteinase-1 (MMP-1). PLoS One 6: e25103, 2011.

29. Kwak JE, Kim HS, Joo M, Chang SH, Shim SH and Lee HR: The expression of galectin-3 and galectin-7 in epithelial dysplasia and adenocarcinoma of the stomach. Korean J Pathol 42: 365-372, 2008.

30. Saito Y, Sekine W, Sano R, et al: Potentiation of cell invasion and matrix metalloproteinase production by $\alpha 3 \beta 1$ integrin-mediated adhesion of gastric carcinoma cells to laminin-5. Clin Exp Metastasis 27: 197-205, 2010.

31. Takatsuki H, Komatsu S, Sano R, Takada Y and Tsuji T: Adhesion of gastric carcinoma cells to peritoneum mediated by

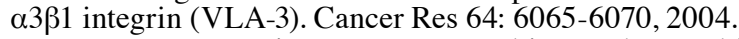

32. Ura H, Denno R, Hirata K, Yamaguchi $K$ and Yasoshima T: Separate functions of $\alpha 2 \beta 1$ and $\alpha 3 \beta 1$ integrins in the metastatic process of human gastric carcinoma. Surg Today 28: 1001-1006, 1998.

33. Adachi M, Taki T, Huang C, et al: Reduced integrin alpha3 expression as a factor of poor prognosis of patients with adenocarcinoma of the lung. J Clin Oncol 16: 1060-1067, 1998.

34. Hashida H, Takabayashi A, Tokuhara T, et al: Integrin $\alpha 3$ expression as a prognostic factor in colon cancer: association with MRP-1/CD9 and KAI1/CD82. Int J Cancer 97: 518-525, 2002.

35. Kurokawa A, Nagata M, Kitamura N, et al: Diagnostic value of integrin $\alpha 3, \beta 4$, and $\beta 5$ gene expression levels for the clinical outcome of tongue squamous cell carcinoma. Cancer 112: 1272-1281, 2008

36. Zhu GH, Huang C, Qiu ZJ, et al: Expression and prognostic significance of CD151, c-Met, and integrin alpha3/alpha6 in pancreatic ductal adenocarcinoma. Dig Dis Sci 56: 1090-1098, 2011. 
37. Park SY, Kook MC, Kim YW, Cho NY, Kim TY and Kang GH: Mixed-type gastric cancer and its association with high-frequency $\mathrm{CpG}$ island hypermethylation. Virchows Arch 456: 625-633, 2010.

38. Alves MK, Ferrasi AC, Lima VP,Ferreira MV, de Moura Campos Pardini MI and Rabenhorst SH: Inactivation of $C O X-2, H M L H I$ and $C D K N 2 A$ genes by promoter methylation in gastric cancer: relationship with histological subtype, tumor location and Helicobacter pylori genotype. Pathobiology 78: 266-276, 2011.

39. Alves MK, Lima VP, Ferrasi AC, Rodrigues MA, De Moura Campos Pardini MI and Rabenhorst SH: $C D K N 2 A$ promoter methylation is related to the tumor location and histological subtype and associated with Helicobacter pylori fla $\mathrm{A}(+)$ strains in gastric adenocarcinomas. APMIS 118: 297-307, 2010.

40. Dong CX, Deng DJ, Pan KF, et al: Promoter methylation of p16 associated with Helicobacter pylori infection in precancerous gastric lesions: a population-based study. Int J Cancer 124 434-439, 2009

41. Kang GH, Lee S, Cho NY, et al: DNA methylation profiles of gastric carcinoma characterized by quantitative DNA methylation analysis. Lab Invest 88: 161-170, 2008.
42. Maekita T, Nakazawa K, Mihara M, et al: High levels of aberrant DNA methylation in Helicobacter pylori-infected gastric mucosae and its possible association with gastric cancer risk. Clin Cancer Res 12: 989-995, 2006.

43. Peterson AJ, Menheniott TR, O'Connor L, et al: Helicobacter pylori infection promotes methylation and silencing of trefoil factor 2, leading to gastric tumor development in mice and humans. Gastroenterology 139: 2005-2017, 2010

44. Essner JJ, Chen E and Ekker SC: Syndecan-2. Int J Biochem Cell Biol 38: 152-156, 2006.

45. Han I, Park H and Oh ES: New insights into syndecan-2 expression and tumourigenic activity in colon carcinoma cells. J Mol Histol 35: 319-326, 2004.

46. Bai F, Guo X, Yang L, et al: Establishment and characterization of a high metastatic potential in the peritoneum for human gastric cancer by orthotopic tumor cell implantation. Dig Dis Sci 52: 1571-1578, 2007.

47. Budarf ML, Goyette P, Boucher G, et al: A targeted association study in systemic lupus erythematosus identifies multiple susceptibility alleles. Genes Immun 12: 51-58, 2011. 